\title{
Optimization of Stormwater Filtration at the Urban/Watershed Interface
}

Hipp, J. A.; Ogunseitan, O. A.; Lejano, R.; Smith, C. S.

This Supporting Information Section includes this cover sheet, five tables (A-E) and one Figure with three panels. Tables A and B detail the filter inserts tested. Table C provides the filter removal efficiencies in table format along with needed removal rates. Table D presents TMDL load allocations and monitored data for San Diego Creek and Newport Bay. Event Mean Concentration levels used for the optimization model are included in Table E. Figure A shows the results of optimization modeling of the location of filters for meeting TMDL limits for TSS (panel a), Cd (panel b), and Zn (panel c). 
Table A. Commercial information on catch basin insert filters.

\begin{tabular}{|c|c|c|c|c|c|c|}
\hline $\begin{array}{l}\text { Filter } \\
\text { Company }\end{array}$ & $\begin{array}{l}\text { Typ } \\
\text { e }\end{array}$ & Insert Material & $\begin{array}{l}\text { Fill } \\
\text { Capacity } \\
\left(\mathrm{cm}^{3}\right)\end{array}$ & $\begin{array}{l}\text { Max. Flow } \\
\text { Rate } \\
\text { (LPM) }\end{array}$ & $\begin{array}{l}\text { Operation and } \\
\text { Maintenance }\end{array}$ & $\begin{array}{l}\text { Cost } \\
(\$)\end{array}$ \\
\hline A1 & Box & $\begin{array}{l}\text { OARS } \\
\text { Smartsponge }\end{array}$ & $N A^{1}$ & 4,164 & NA & NA \\
\hline $\mathrm{A} 2$ & Box & $N A^{1}$ & NA & NA & NA & NA \\
\hline B & Box & $\begin{array}{l}\text { Oil absorbing } \\
\text { polymer }^{2}\end{array}$ & 226,535 & 25,381 & $\begin{array}{l}5 \text { year warranty on } \\
\text { product; routine } \\
\text { maintenance and } \\
\text { vacuuming }\end{array}$ & 595 \\
\hline C & $\begin{array}{l}\text { Soc } \\
\mathrm{k}\end{array}$ & $\begin{array}{l}\text { Hydrophobic } \\
\text { absorbent }^{2}\end{array}$ & 2,950 & 1,893 & $\begin{array}{l}\text { Clean, replace every } \\
3-6 \text { mos. }\end{array}$ & 55 \\
\hline $\mathrm{D} 1^{3,4}$ & Box & $\begin{array}{l}\text { Perlite and } \\
\text { HEG- } 150^{5}\end{array}$ & 17,747 & NA & $\begin{array}{l}\text { Replace every 6-12 } \\
\text { mos. }\end{array}$ & NA \\
\hline $\mathrm{D} 2^{3,4}$ & Box & $\begin{array}{l}\text { Perlite and } \\
\text { Highly } \\
\text { Reactive } \\
\text { Carbon } \\
\text { Mixture }^{5}\end{array}$ & 17,747 & NA & $\begin{array}{l}\text { Replace every 6-12 } \\
\text { mos. }\end{array}$ & NA \\
\hline$E$ & Box & Xsorb & $37,707^{6}$ & $\begin{array}{l}1,908- \\
2,362\end{array}$ & $\begin{array}{l}3 \text { cleanings per yr. } \\
\text { (\$65-80/cleaning) }\end{array}$ & $\begin{array}{l}248- \\
276\end{array}$ \\
\hline $\mathrm{F}^{3}$ & Box & Zeolite & $\begin{array}{l}\text { "Built to } \\
\text { suit" }\end{array}$ & 1,893 & $\begin{array}{l}\text { Replace materials } \\
\text { annually and body } \\
\text { every } 10 y \text { rs. }\end{array}$ & $\begin{array}{l}\$ 21.6 \\
7 / \mathrm{kg} \\
\text { of } \\
\text { materi } \\
\text { al }\end{array}$ \\
\hline$G(1 \& 2)^{4}$ & $\begin{array}{l}\text { Soc } \\
k\end{array}$ & $\begin{array}{l}\text { Polypropylene } \\
\text { geotextile }\end{array}$ & 22,614 & $\begin{array}{l}1,893- \\
2,915\end{array}$ & $\begin{array}{l}\text { Clean, replace every } \\
3-6 \text { mos. }\end{array}$ & $55-84$ \\
\hline
\end{tabular}


Table B. Measured descriptors of catch basin insert filters.

\begin{tabular}{|c|c|c|c|c|c|c|c|c|}
\hline $\begin{array}{l}\text { Filter } \\
\text { Company }\end{array}$ & $\begin{array}{l}\text { Influent } \\
\text { Opening } \\
\left(\mathrm{cm}^{2}\right)\end{array}$ & $\begin{array}{l}\text { Total Fill } \\
\text { Capacity } \\
\left(\mathrm{cm}^{3}\right)\end{array}$ & $\begin{array}{l}\text { Media } \\
\text { Volume } \\
\left(\mathrm{cm}^{3}\right)\end{array}$ & $\begin{array}{l}\text { Effluent } \\
\text { Opening } \\
\left(\mathrm{cm}^{2}\right)\end{array}$ & $\begin{array}{l}\text { \% of } \\
\text { Media in } \\
\text { Contact } \\
\text { w/ } \\
\text { Influent }\end{array}$ & $\begin{array}{l}\text { Saturation } \\
\text { Level }(L)^{2}\end{array}$ & $\begin{array}{l}\text { Hydraulic } \\
\text { Loading } \\
\text { Rate } \\
\left(\mathrm{m}^{3} / \mathrm{s} / \mathrm{m}^{2}\right)\end{array}$ & $\begin{array}{l}\text { Initial } \\
\text { Effluent } \\
\text { Color and } \\
\text { Sediment- } \\
\text { ation }\end{array}$ \\
\hline $\mathrm{A} 1$ & 1,048 & $55,921^{1}$ & 16,928 & 1,048 & 85 & $1.1-2.0$ & $4.8 e-5$ & Clear \\
\hline A2 & 1,048 & $55,921^{1}$ & 16,928 & 1,048 & 85 & 2.0 & $4.8 e-5$ & $\begin{array}{l}\text { Slightly clouc } \\
\text { and frothy }\end{array}$ \\
\hline \multirow[t]{2}{*}{ B } & \multirow[t]{2}{*}{3,716} & \multirow[t]{2}{*}{226,535} & $\begin{array}{l}\text { Poly } \\
\text { sock - } \\
2,894\end{array}$ & \multirow[t]{2}{*}{3,716} & $\begin{array}{l}\text { Poly } \\
\text { sock - } \\
25\end{array}$ & \multirow[t]{2}{*}{$1.1-1.2$} & \multirow[t]{2}{*}{$1.3 e-5$} & \multirow[t]{2}{*}{$\begin{array}{l}\text { Black effluer } \\
\text { due to } \\
\text { charcoal } \\
\text { leaching }\end{array}$} \\
\hline & & & $\begin{array}{l}\text { Charcoa } \\
\text { l bag }^{3}- \\
541\end{array}$ & & $\begin{array}{l}\text { Charcoal } \\
\text { bag }^{3}- \\
100\end{array}$ & & & \\
\hline \multirow[t]{2}{*}{ C } & \multirow[t]{2}{*}{11,148} & \multirow[t]{2}{*}{$\begin{array}{l}\text { Sock - } \\
2,950\end{array}$} & $\begin{array}{l}\text { Apron - } \\
11,148 \\
\mathrm{~cm}^{2}\end{array}$ & \multirow[t]{2}{*}{$306^{4}$} & $\begin{array}{l}\text { Apron - } \\
20\end{array}$ & \multirow[t]{2}{*}{1.1} & \multirow[t]{2}{*}{$4.5 e-6$} & \multirow[t]{2}{*}{$\begin{array}{l}\text { Slightly froth } \\
\text { but clear }\end{array}$} \\
\hline & & & $\begin{array}{l}\text { Sock - } \\
1,990 \\
\mathrm{~cm}^{2}\end{array}$ & & $\begin{array}{l}\text { Sock - } \\
100\end{array}$ & & & \\
\hline D1 & 1,703 & $75,512^{1}$ & 23,597 & $4^{5}$ & 100 & $4.5-9.0$ & $2.9 e-5$ & Clear \\
\hline $\mathrm{D} 2$ & 1,703 & $75,512^{1}$ & 23,597 & $4^{5}$ & 100 & $3.0-6.0$ & $2.9 e-5$ & Slightly gray \\
\hline E & 2,768 & 63,270 & 7,571 & 2,265 & 25 & 1.1 & $1.8 \mathrm{e}-5$ & $\begin{array}{l}\text { Mostly clear, } \\
\text { few media } \\
\text { dust particle }\end{array}$ \\
\hline $\mathrm{F}$ & 1,950 & $42,482^{1}$ & 42,482 & 1950 & 85 & $2.0-4.0$ & $2.6 e-5$ & $\begin{array}{l}\text { Brown to } \\
\text { black } \\
\text { discoloration } \\
\text { and } \\
\text { sedimentatio }\end{array}$ \\
\hline \multirow[t]{3}{*}{ G1 } & \multirow[t]{3}{*}{8,361} & \multirow[t]{3}{*}{$\begin{array}{l}\text { Sock - } \\
7,217\end{array}$} & $\begin{array}{l}\text { Apron - } \\
8,361 \\
\mathrm{~cm}^{2}\end{array}$ & \multirow[t]{3}{*}{$170^{4}$} & $\begin{array}{l}\text { Apron - } \\
20\end{array}$ & \multirow[t]{3}{*}{$1.1-1.5$} & \multirow[t]{3}{*}{$6.0 e-6$} & \multirow[t]{3}{*}{ Clear } \\
\hline & & & $\begin{array}{l}\text { Sock - } \\
1,793 \\
\mathrm{~cm}^{2}\end{array}$ & & $\begin{array}{l}\text { Sock - } \\
100\end{array}$ & & & \\
\hline & & & Filler - & & & & & \\
\hline
\end{tabular}


$847 \mathrm{~cm}^{2}$

G2

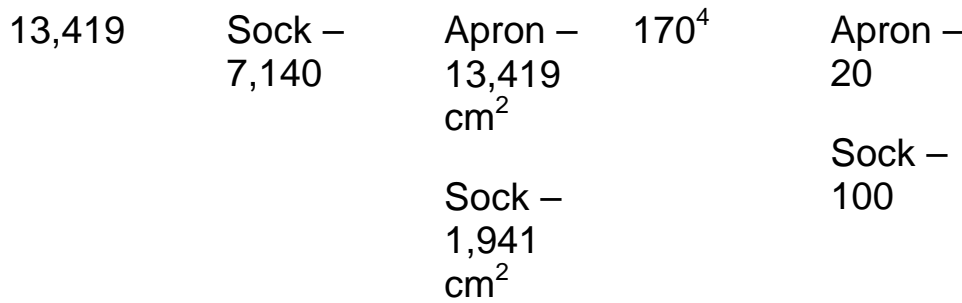

${ }^{1}$ Total Fill Capacity for A, D, \& F filters includes media area. Other filters are either sock filters or their media are essential flat sheets. ${ }^{2}$ Amount of non-contaminated influent water poured through gutter and insert filter to receive a minimum of $1 \mathrm{~L}$ of effluent. Range due to multiple tests and varying thickness of insert media. ${ }^{3}$ Multiple charcoal bags can be used. ${ }^{4}$ Effluent opening of the two sock filters includes only the exit holes in the cloth and does not include water leaching through the actual material. ${ }^{5}$ Consists of three $1.3 \mathrm{~cm}$ diameter circles. 
Table C. Filter effectiveness (\%) for each pollutant and necessary removal proportions (Highest monitored data/TMDL). Mean pollutant removal effectiveness (\%) is represented by top number and the standard deviation is in parenthesis. Bold, italicized numbers meet essential pollutant removal effectiveness.

\section{Pollutant}

\begin{tabular}{|c|c|c|c|c|c|c|c|}
\hline $\begin{array}{l}\text { Filter } \\
\text { Company }\end{array}$ & Cd & $\mathrm{Cu}$ & $\mathrm{Pb}$ & $\mathrm{Se}$ & $\mathrm{Zn}$ & Coliform & TSS \\
\hline A1 & $\begin{array}{l}18.2 \\
(25.7)\end{array}$ & $\begin{array}{l}17.9 \\
(25.3)\end{array}$ & $\begin{array}{l}63.9 \\
(5.6)\end{array}$ & $\begin{array}{l}8.9 \\
(12.6)\end{array}$ & $\begin{array}{l}8.4 \\
(6.4)\end{array}$ & $\begin{array}{l}25.0 \\
(28.5)\end{array}$ & $\begin{array}{l}82.1 \\
(7.2)\end{array}$ \\
\hline A2 & $92.8(2.7)$ & $\begin{array}{l}17.5 \\
(24.8)\end{array}$ & $\begin{array}{l}100.0 \\
(0.0)\end{array}$ & $\begin{array}{l}85.2 \\
(6.5)\end{array}$ & $\begin{array}{l}0.0 \\
(0.0)\end{array}$ & $0.0(0.0)$ & $\begin{array}{l}80.1 \\
(15.8)\end{array}$ \\
\hline B & $94.7(7.4)$ & $\begin{array}{l}7.1 \\
(10.1)\end{array}$ & $\begin{array}{l}98.8 \\
(1.7)\end{array}$ & $\begin{array}{l}8.9 \\
(4.8)\end{array}$ & $\begin{array}{l}11.0 \\
(8.1)\end{array}$ & $\begin{array}{l}32.8 \\
(24.0)\end{array}$ & $\begin{array}{l}53.0 \\
(26.9)\end{array}$ \\
\hline C & $8.5(0.9)$ & $\begin{array}{l}22.5 \\
(3.5)\end{array}$ & $\begin{array}{l}26.4 \\
(0.3)\end{array}$ & $\begin{array}{l}1.8 \\
(2.5)\end{array}$ & $\begin{array}{l}0.1 \\
(0.2)\end{array}$ & $\begin{array}{l}23.8 \\
(18.5)\end{array}$ & $\begin{array}{l}93.9 \\
(2.5)\end{array}$ \\
\hline D1 & $84.9(8.5)$ & $\begin{array}{l}24.3 \\
(29.3)\end{array}$ & $\begin{array}{l}100.0 \\
(0.0)\end{array}$ & $\begin{array}{l}59.3 \\
(33.7)\end{array}$ & $\begin{array}{l}71.7 \\
(2.1)\end{array}$ & $\begin{array}{l}22.4 \\
(29.5)\end{array}$ & $\begin{array}{l}62.0 \\
(21.7)\end{array}$ \\
\hline D2 & $77.2(6.3)$ & $\begin{array}{l}26.4 \\
(23.2)\end{array}$ & $\begin{array}{l}100.0 \\
(0.0)\end{array}$ & $\begin{array}{l}33.9 \\
(1.8)\end{array}$ & $\begin{array}{l}74.8 \\
(1.6)\end{array}$ & $\begin{array}{l}39.3 \\
(33.2)\end{array}$ & $\begin{array}{l}63.4 \\
(26.5)\end{array}$ \\
\hline E & $\begin{array}{l}22.5 \\
(13.2)\end{array}$ & $\begin{array}{l}0.0 \\
(0.0)\end{array}$ & $\begin{array}{l}94.6 \\
(2.6)\end{array}$ & $\begin{array}{l}15.3 \\
(21.7)\end{array}$ & $\begin{array}{l}19.8 \\
(9.6)\end{array}$ & $\begin{array}{l}20.3 \\
(16.9)\end{array}$ & $\begin{array}{l}94.9 \\
(2.6)\end{array}$ \\
\hline$F$ & $94.7(7.4)$ & $\begin{array}{l}0.0 \\
(0.0)\end{array}$ & $\begin{array}{l}21.4 \\
(50.0)\end{array}$ & $\begin{array}{l}43.2 \\
(0.5)\end{array}$ & $\begin{array}{l}47.5 \\
(18.6)\end{array}$ & $\begin{array}{l}46.6 \\
(32.4)\end{array}$ & $\begin{array}{l}0.0 \\
(0.0)\end{array}$ \\
\hline G1 & $\begin{array}{l}47.6 \\
(22.7)\end{array}$ & $\begin{array}{l}54.3 \\
(1.0)\end{array}$ & $\begin{array}{l}81.6 \\
(26.1)\end{array}$ & $\begin{array}{l}9.2 \\
(13.0)\end{array}$ & $\begin{array}{l}42.3 \\
(15.0)\end{array}$ & - & $\begin{array}{l}78.7 \\
(15.9)\end{array}$ \\
\hline G2 & - & - & - & - & - & $\begin{array}{l}10.7 \\
(8.5)\end{array}$ & $\begin{array}{l}74.5 \\
(33.2)\end{array}$ \\
\hline $\begin{array}{l}\text { Mean } \\
\text { Removal }\end{array}$ & $\begin{array}{l}60.1 \\
(36.0)\end{array}$ & $\begin{array}{l}18.9 \\
(16.6)\end{array}$ & $\begin{array}{l}76.3 \\
(32.0)\end{array}$ & $\begin{array}{l}29.5 \\
(28.4)\end{array}$ & $\begin{array}{l}30.6 \\
(29.4)\end{array}$ & $\begin{array}{l}24.5 \\
(14.1)\end{array}$ & $\begin{array}{l}68.3 \\
(27.5)\end{array}$ \\
\hline $\begin{array}{l}\text { Needed } \\
\text { removal }\end{array}$ & 50.6 & 87.9 & - & 69.0 & 55.7 & 98.3 & 50.0 \\
\hline
\end{tabular}


Table D. TMDL Load Allocation and monitored data for San Diego Creek and Newport Bay.

\begin{tabular}{|c|c|c|c|c|c|c|}
\hline Pollutant & $\begin{array}{l}\text { Base } \\
\text { Flow } \\
\text { Acute } \\
\text { TMDL }\end{array}$ & $\begin{array}{l}\text { Large } \\
\text { Flow } \\
\text { Acute } \\
\text { TMDL }\end{array}$ & $\begin{array}{l}\text { Dissolved } \\
\text { Saltwater } \\
\text { Acute } \\
\text { TMDL }\end{array}$ & $\begin{array}{l}\text { Geometric } \\
\text { mean ( } 5 \\
\text { sample } \\
\text { avg. over } \\
30 \text { days) }\end{array}$ & $\begin{array}{l}\text { Monitored } \\
\text { Data - } \\
\text { San Diego } \\
\text { Creek }\end{array}$ & $\begin{array}{l}\text { Monitored } \\
\text { Data - } \\
\text { Newport } \\
\text { Bay }\end{array}$ \\
\hline $\operatorname{Se}(\mu \mathrm{g} / \mathrm{L})$ & 20.0 & 20.0 & 71.0 & - & 64.6 & - \\
\hline $\mathrm{Cd}(\mu \mathrm{g} / \mathrm{L})$ & 19.1 & 8.9 & 42.0 & - & 18.0 & 2.0 \\
\hline $\mathrm{Cu}(\mu \mathrm{g} / \mathrm{L})$ & 50.0 & 25.5 & 4.8 & - & 210.0 & 24.0 \\
\hline $\mathrm{Pb}(\mu \mathrm{g} / \mathrm{L})$ & 281.0 & 134.0 & 210.0 & - & 46.0 & 4.0 \\
\hline $\mathrm{Zn}(\mu \mathrm{g} / \mathrm{L})$ & 379.0 & 208.0 & 90.0 & - & 470.0 & 59.0 \\
\hline $\begin{array}{l}\text { Fecal } \\
\text { Coliform }\end{array}$ & - & - & 4 & 2 & - & $\begin{array}{l}25 \\
\text { (Geomean) }\end{array}$ \\
\hline (CFU/ml) & & & & & & 230 (Acute) \\
\hline
\end{tabular}


Table E. Event Mean Concentrations (EMC) used for estimating stormwater pollutant loadings.

\begin{tabular}{|c|c|c|c|c|c|c|c|}
\hline Pollutant & Vacant & $\begin{array}{l}\text { Modera } \\
\text { te } \\
\text { Density } \\
\text { Resid- } \\
\text { ential }\end{array}$ & $\begin{array}{l}\text { Multi-Family } \\
\text { Residential }\end{array}$ & $\begin{array}{l}\text { Mixed } \\
\text { Residential }\end{array}$ & Industrial & $\begin{array}{l}\text { Trans- } \\
\text { portation }\end{array}$ & $\begin{array}{l}\text { Retail / } \\
\text { Comm- } \\
\text { ercial }\end{array}$ \\
\hline $\mathrm{Cd}(\mu \mathrm{g} / \mathrm{L})$ & 0.6 & 0.6 & 0.6 & 0.5 & 0.5 & 0.5 & 0.7 \\
\hline $\mathrm{Zn}(\mu \mathrm{g} / \mathrm{L})$ & 120 & 150 & 150 & 120 & 360 & 276 & 197 \\
\hline $\begin{array}{l}\text { TSS } \\
(\mathrm{mg} / \mathrm{L})\end{array}$ & 192 & 40 & 40 & 62 & 157 & 64 & 70 \\
\hline $\begin{array}{l}\text { Percent } \\
\text { Impervious }\end{array}$ & 10 & 55 & 74 & 59 & 91 & 91 & 90 \\
\hline
\end{tabular}

Event mean concentration and percent impervious values adapted from LADPW. 1999. Los Angeles County Stormwater Monitoring Report: 1998-1999. Los Angeles: Los Angeles Department of Public Works; and Schiff, Kenneth. 1996. Review of Existing Stormwater Monitoring Programs for Estimating Bight-wide Mass Emissions from Urban Runoff: Southern California Coastal Water Research Project. 


\section{A}

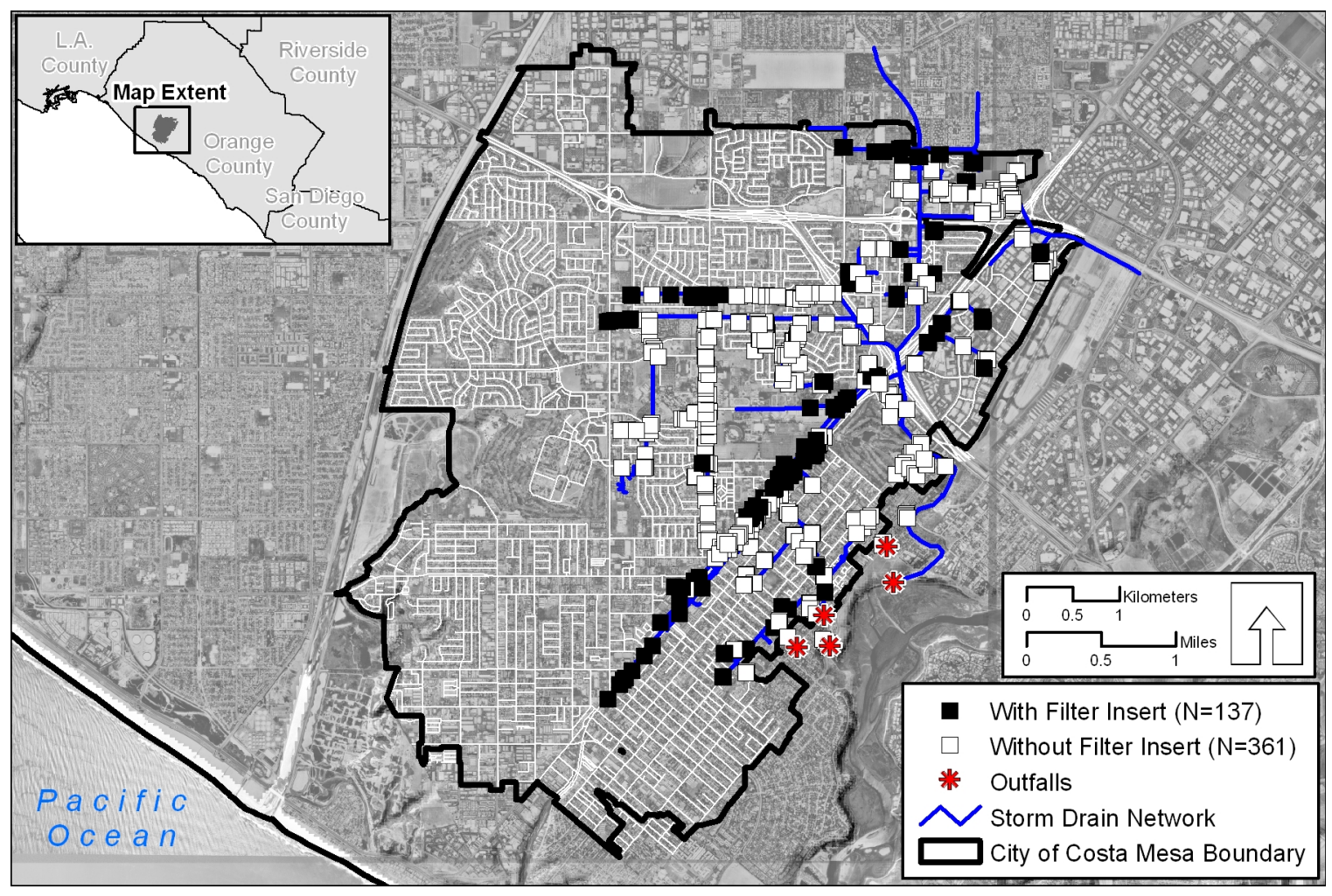

B

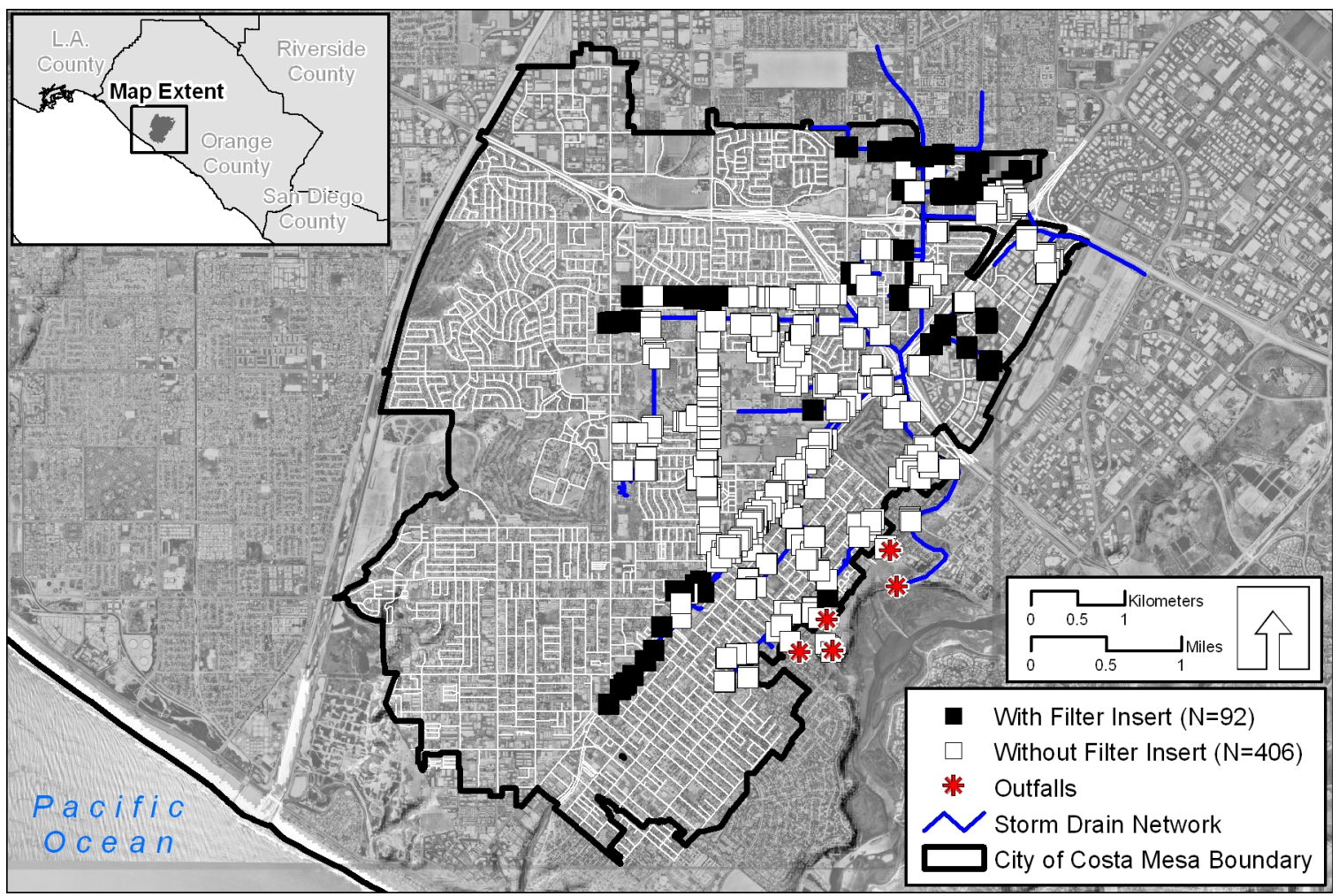


C

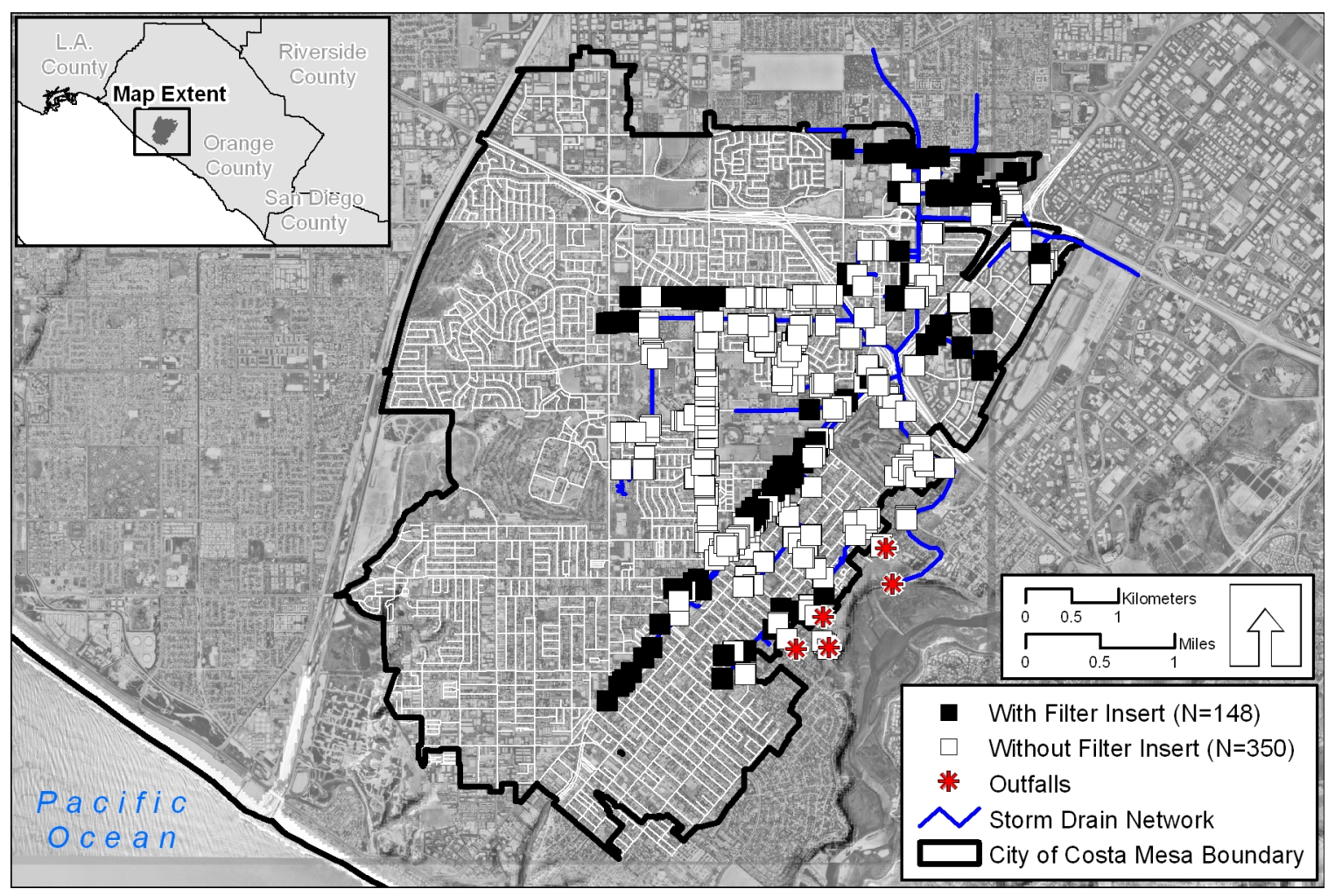

Figure A. Panel a: Optimized Placement of Filter Inserts by Curb Inlet, TSS TMDL Compliance. Panel b: Optimized Placement of Filter Inserts by Curb Inlet, Cd TMDL Compliance. Panel c: Optimized Placement of Filter Inserts by Curb Inlet, Zn TMDL Compliance. 